Théologiques

Théologiques

\title{
L'anthropologue et la dualité paradoxale du " croire " occidental
}

\section{Roberte N. Hamayon}

Volume 13, numéro 1, printemps 2005

Croire et croyances

URI : https://id.erudit.org/iderudit/012523ar

DOI : https://doi.org/10.7202/012523ar

Aller au sommaire du numéro

\section{Éditeur(s)}

Faculté de théologie et de sciences des religions, Université de Montréal

\section{ISSN}

1188-7109 (imprimé)

1492-1413 (numérique)

Découvrir la revue

Citer cet article

Hamayon, R. N. (2005). L'anthropologue et la dualité paradoxale du « croire " occidental. Théologiques, 13(1), 15-41. https://doi.org/10.7202/012523ar
Résumé de l'article

Un embarras certain entoure l'usage des termes croire et croyance dans les travaux des anthropologues. L'argument développé ici porte sur l'une des causes de cet embarras : le double sens qu'ont ces termes dans les langues occidentales sous l'influence du christianisme et qui semble caractéristique des religions universalistes. Ce double sens révèle un lien implicite entre objet de croyance et attitude de croyance, la primauté logique revenant à l'attitude et l'objet lui étant subordonné. Il peut en découler que l'objet acquière un statut de Vérité et que l'attitude devienne radicale et systématique, détachée de toute confrontation avec la réalité.

L'exemple de la terminologie mongole illustre, par contraste, l'absence d'un tel lien - absence qui caractérise, semble-t-il, un grand nombre de cultures non-occidentales. En effet, les verbes mongols que l'on peut traduire par " croire » s'appliquent exclusivement à l'attitude de croyance, que les Mongols envisagent éventuellement dans son prolongement, c'est-à-dire l'action rituelle à laquelle elle donne lieu. L'absence de renvoi à des objets de croyance donnés apparaît comme une source de souplesse et d'innovation. Elle engendre une dynamique spéculative volontariste qui s'exprime surtout par les procédures divinatoires.
Tous droits réservés @ Faculté de théologie et de sciences des religions, Université de Montréal, 2006
Ce document est protégé par la loi sur le droit d'auteur. L'utilisation des services d'Érudit (y compris la reproduction) est assujettie à sa politique d'utilisation que vous pouvez consulter en ligne.

https://apropos.erudit.org/fr/usagers/politique-dutilisation/ 


\title{
L'anthropologue et la dualité paradoxale du « croire » occidental ${ }^{1}$
}

\author{
Roberte N. Hamayon \\ École Pratique des Hautes Études (ÉPHÉ) \\ Sorbonne, Paris
}

\section{L'usage paradoxal du terme croyance dans les langues occidentales}

Le terme croyance constitue, pour les anthropologues, depuis les débuts de leur discipline, un problème épistémologique tel que nombreux sont ceux d'entre eux qui n'osent plus l'utiliser. Je commencerai par rappeler brièvement, et à seule fin d'introduire mon propos, les raisons de leur réticence en évoquant les positions de certains des auteurs qui les ont analysées. J'aimerais ensuite tenter de reformuler la principale de ces raisons, qui est la polysémie de ce terme.

Dans un texte fondateur qu'il lui consacre, Jean Pouillon constate que «croire à » n'a pas le même sens que «croire en », et que l'un et l'autre diffèrent de «croire + objet direct» et «croire que ». Sa réflexion l'amène à conclure que «la polysémie du "croire" est irréductible» $(1993,21)$. Cette notion, écrit-il, «ne renvoie à aucun sens privilégié » et "consiste en une liaison paradoxale de sens contradictoires» (29-30), ce qui explique que "le doute accompagne la croyance» (26). Je voudrais toutefois m'en tenir ici à ce qui me semble constituer le paradoxe inscrit au cœur de cette notion, dans l'usage que nous en faisons en Occident. En effet, nous appliquons le terme croyance aussi bien à ce que l'on croit qu'au fait même de croire, à un contenu idéel qu'à l'adhésion à ce contenu; nous parlons aussi bien de «croyances » pour évoquer des conceptions religieuses — et l'emploi du

1. Je remercie vivement tous ceux qui ont nourri mon travail sur ce thème par leurs stimulantes questions et suggestions: Robert Crépeau, initiateur du colloque "Religion: croire et croyances " tenu à Montréal le 6 février 2005, Jean-Paul Willaime, coresponsable d'un séminaire tenu à l'ÉPHÉ en 2002, les auditeurs de ce séminaire, Grégoire Schlemmer, et les évaluateurs de la revue Théologiques. 
pluriel est alors significatif — que de «la croyance» comme état d'esprit, comme attitude mentale, psychique ou affective du sujet croyant ${ }^{2}$. Et c'est cette dualité de sens qui est source de paradoxe. Or, si elle est présente dans les langues dans lesquelles ont été écrits la plupart des travaux anthropologiques, celles de l'Occident judéo-chrétien, elle ne l'est pas dans la plupart des langues des peuples non-occidentaux qui en sont l'objet. On pourrait donc l'ignorer là où elle n'est pas exprimée, pour s'interroger séparément sur ce que ces peuples «croient » et sur le fait qu'ils «croient », et se demander laquelle de ces questions présente le plus d'intérêt pour une réflexion anthropologique. Mais on peut aussi choisir de raisonner à partir de cette dualité paradoxale: peut-être y a-t-il là un moyen de jeter un éclairage sur les implications respectives de sa présence ou de son absence dans une culture religieuse et de féconder le questionnement sur le rapport entre les deux sens de notre notion de croyance. C'est donc à un cas concret de vocabulaire ne faisant pas de lien entre ces deux sens que je m'attacherai pour finir, celui des verbes mongols pouvant se traduire par «croire».

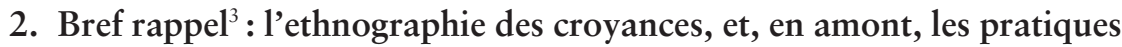

En amont de ce questionnement, un autre facteur a imprimé sa marque sur l'ethnologie occidentale à l'époque coloniale: le caractère éminemment ritualiste et non-doctrinal de bon nombre des formes religieuses observées sur les terrains lointains, notamment dans les sociétés sans écriture. L'exécution des actes rituels y est la caution par excellence du respect de la religion: il suffit d'accomplir les gestes prescrits, de prononcer les formules prescrites. Ainsi, la pratique effective tient lieu d'attestation de croyance,

2. La distinction recoupe donc à peu près la répartition des usages du terme « croyance » entre le pluriel et le singulier, ou encore entre " les croyances » et "le croire ». Cependant, le courant contemporain de sociologie des religions qui a propagé cet usage nominal du verbe en français l'emploie souvent dans une perspective qui déborde le fait d'adhérer à une croyance et englobe l'ensemble du fait religieux (Hervieu-Léger 1997), ce qui montre à quel point il est difficile de maintenir une distinction entre ces deux sens. Je remercie l'évaluateur de m'indiquer que déjà Michel de Certeau (1987) avait montré que la croyance était une modalité d'affirmation et non le simple objet du croire.

Par convention, j'utiliserai ici le pluriel là où il pourra faciliter l'acception du sens : contenu de croyance.

3. Ce rappel introductif laisse, hélas, dans l'ombre de nombreux travaux pertinents pour les questions abordées ici. 
sans qu'il y ait besoin de manifestation formelle d'adhésion. Si, pour certains auteurs, l'acte rituel, machinal et répétitif, est par lui-même vide de sens, pour d'autres, il est l'expression de croyances qui en sont le fondement conceptuel et qu'il leur revient de retracer ${ }^{4}$. L'attention porte dès lors conjointement sur les rites et les croyances sous-jacentes - ce qui longtemps a valu au sens d'objet ou de contenu de croyance une certaine prééminence dans les emplois de ce terme en anthropologie.

Par ailleurs, l'évidence du caractère collectif et récurrent des pratiques au sein d'une société donnée impose l'idée que les croyances sous-jacentes forment toutes ensemble le contenu conceptuel de la religion de cette société et qu'elles sont partagées par ses membres. Une certaine cohérence interne des croyances au sein de toute société est un postulat de base de la sociologie des religions dès son origine. Elle est au cœur de l'œuvre de Durkheim ${ }^{5}$ et règne toujours aujourd'hui à travers l'usage de la notion de système pour parler des faits religieux, quelque nom qu'on leur donne, et que l'on conçoive ce système comme simple ou complexe, ouvert ou fermét.

\section{Seuls les autres ont des «croyances » et elles sont «fausses »}

Cependant, pour nombre d'observateurs occidentaux de l'époque coloniale, les «croyances» des sociétés qu'ils observent ne peuvent être que «fausses » puisqu'elles diffèrent de ce qu'eux-mêmes pensent — et qu'ils n'appellent d'ailleurs pas «croyances ». Ou si l'on préfère, le seul fait d'appeler «croyances » les idées que l'on ne partage pas, les idées des autres donc, revient implicitement à décréter à la fois que ce sont des idées «fausses » et que ces autres y «croient » — ce qui démontre à quel point leur culture est

4. Pour une discussion originale du rapport au «sens » de l'acte rituel, voir Pitt-Rivers 1984-1985. Pour les rapports entre rite et croyance et une définition du ritualisme, voir Héran 1986 et Linder et Scheid 1993.

5. Rappelons l'essentiel de sa définition: «Une religion est un système solidaire de croyances et de pratiques relatives à des choses sacrées [...], croyances et pratiques qui unissent en une même communauté morale [...] tous ceux qui y adhèrent" (Durkheim 1960, 65). Telle sera aussi la position d'un Mauss ( "Il n'y a pas, en fait, une chose, une essence, appelée Religion : il n’y a que des phénomènes religieux plus ou moins agrégés en des systèmes [...] qui ont une existence historique définie. » $[1968,93])$, d'un Radcliffe-Brown et d'autres à leur suite.

6. Ainsi le $\mathrm{xx}^{\mathrm{e}}$ siècle a-t-il vu se succéder "système de croyances [et de pratiques]", «système de représentations et de pratiques », "système de pensée » ou encore «système symbolique". 
«irrationnelle», étant a priori hors de question qu'ils puissent ne pas «croire » à leurs «croyances".

Peut-on en rester là ? D'un côté, l'idée prévaut longtemps que peu importe que l'indigène soit dans l'erreur, l'ethnologue n'est pas un missionnaire et n'a pas à le convertir — d'ailleurs, le missionnaire parlerait plutôt de superstition. Du reste, le folkloriste ne relève-t-il pas, lui aussi, aux marges du christianisme, dans le culte des saints par exemple, des «croyances populaires » que les tenants les plus stricts du dogme qualifient de superstitions? Pour le chrétien, le dogme auquel il adhère n'est pas lui-même une croyance et peut même être défini par opposition à toutes les croyances ${ }^{7}$.

Mais d'un autre côté, il y a toujours, dans l'usage même du terme croyance, une sorte de sous-entendu qui embarrasse. Si des "croyances» sont partagées au sein d'une société au point d'y former système et d'y fonctionner comme une religion, peut-on en rendre compte en les appréhendant comme des idées fausses auxquelles les membres de cette société adhèrent? Et la question de la vérité ou de la fausseté des «croyances» ne perd-elle pas toute pertinence dès lors que l'on prend conscience que chaque société a les siennes, qui diffèrent de celles des autres?

\section{Les « croyances » comme "représentations » : caractère culturel et relativité, caractère systématique et validité}

Durkheim, à nouveau, a magistralement répondu à cet embarras en promouvant la notion de représentation, terme sans doute à ses yeux si consensuel, remarque William S.F. Pickering, qu'il n'a pas trouvé utile de le définir (Pickering $\left.{ }^{8} 2000,12\right)$. Ce terme semble en effet à la fois objectif et neutre,

7. En effet, on ne parle pas de « croyances chrétiennes », mais éventuellement, de croyances du christianisme populaire. De même, on ne dira pas que l'on croit en les saints de la même manière que l'on croit en Dieu.

8. Cet auteur fait état de l'impossibilité de trouver dans l'anglais contemporain un équivalent du français «représentation» tel qu'il est utilisé par Durkheim (Pickering 2000, xiv).

Cependant, à l'époque médiévale, les usages anglais et français du verbe représenter étaient très proches. Dans une étude qu'il consacre à cette notion, Daniel de Coppet traduit la définition qu'en donne R. Williams (Keywords. A Vocabulary of Culture and Society, Glasgow, Fontana, 1976, p. 223-223) : "To represent signifiait "rendre présent physiquement, soi-même ou un autre en personne à quelqu'un [...]; mais aussi à l'esprit [par des histoires], et aux yeux par des peintures [...] ou par des scènes jouées [...]. Mais au $14^{\mathrm{e}}$ siècle il y eut une extension cruciale du sens lorsque le mot 
dénué en lui-même de jugement de valeur en termes de véracité et de présupposé sur l'adhésion. Durkheim n'élude pas pour autant cet aspect de la question.

Une représentation collective, parce qu'elle est collective, présente déjà des garanties d'objectivité; car ce n'est pas sans raison qu'elle a pu se généraliser et se maintenir avec une suffisante persistance. [..., elle] est nécessairement soumise à un contrôle indéfiniment répété: les hommes qui y adhèrent la vérifient par leur expérience propre. (Durkheim 1960, 625)

Le recours à l'expression "représentation collective » permettait d'en mettre le caractère culturel au premier plan et de rendre compte, par là même, de la relativité des croyances: toute société a son propre système de croyances, qui fonctionne en son sein et que chacun de ses membres met en pratique. Cette position a été longtemps perçue comme une garantie d'équité et de scientificité, tout en contribuant à justifier de négliger l'embarrassante question de l'attitude de croyance. Le constat que chaque société a ses propres croyances ne rend-il pas futile de s'interroger sur ce que ressentent les membres d'une société donnée lorsqu'ils accomplissent les gestes rituels correspondants? L'appartenance à ladite société n'est-elle pas une raison suffisante de partager ses croyances comme ses pratiques? N'est-ce pas ce que soutiennent les informateurs eux-mêmes, si nombreux à répondre aux ethnologues qui les interrogent: «c'est ainsi que faisaient nos ancêtres, c'est notre tradition » ? Réponse qui implique qu'il n'y a pas d'autre explication à chercher, puisque, de toute façon, chacun est convaincu à la fois que, s'il est aujourd'hui vivant, c'est que ses ancêtres ont bien fait d'agir comme ils l'ont fait, et que quiconque a d'autres ancêtres agit autrement. En réalité, beaucoup d'attitudes sont possibles non seulement en deçà d'une véritable

"représenter" fut employé pour "symboliser" ou pour "tenir lieu de" ». «Dans un français plus ancien, poursuit Coppet, "représentation" était une construction particulière [...] qui avait la vertu de rendre à nowveau présent» (1992, 120). L'auteur expose ensuite les diverses « constructions particulières » utilisées lors des funérailles d'un roi pour « rendre présent » le pouvoir royal en attendant l'investiture d'un nouveau roi.

La notion de représentation me semble par ailleurs avoir l'intérêt de fournir un accès intéressant à la fonction symbolique, pour autant que "représenter " consiste, dans son principe, à " rendre présent » ce qui ne peut ou ne doit pas l'être : ce qui est représenté est donc nécessairement distinct de ce qui représente (voir Hamayon 1997). Cette distinction est une condition de la fonction symbolique. Les représentations relèvent d'un registre spécifique, par définition distinct de celui de l'analyse. Objet de l'analyse, elles ne peuvent constituer le discours de l'analyse. 
adhésion, mais même sans adhésion du tout, à commencer par le simple conformisme ou la crainte d'une sanction pour non-observation d'une pratique courantes.

Pour les peuples intéressés, la référence à l'héritage des ancêtres ou à la coutume comme fondement des «croyances" peut suffire à justifier de les mettre en acte sans en contester la teneur. Sous ce jour, les croyances sont des choix qui n'ont pas à être justifiés ni discutés, pour autant que le simple fait d'appartenir à une société entraîne d'en assumer les choix. De surcroît, c'est bien ce partage des croyances qui est «l'armature d'une société » et, pour que les choses y soient en ordre, «il suffit donc de faire comme si [...]» (Pouillon 1993, 24). C'est là un point de vue qui, toute logique analytique mise à part, convient particulièrement bien à la posture globalement agnostique qui prévaut dans l'anthropologie française au fil du $\mathrm{Xx}^{\mathrm{e}}$ siècle. Il rejoint celui qu'avait développé Claude Lévi-Strauss à propos de l'adolescent zuni qui, accusé de sorcellerie, finit par s'avouer coupable et produire les preuves de sa culpabilité pour échapper à la condamnation: mieux valait, remarque l'auteur, un système magique que pas de système du tout; tout le monde était rassuré. Le partage des croyances était la source du consensus social ${ }^{10}$ (Lévi-Strauss 1957, 189-193).

En somme, pour ces auteurs, l'organisation des croyances en système au sein d'une société donnée suffit à rendre compte de l'existence d'un consensus à leur sujet et de la fonction de cohésion sociale qu'elles y remplissent. Elle explique que ces "croyances ", comprises comme des choix culturels, puissent être valides au sein de cette société, sans que se pose la question de leur vérité ou de leur fausseté ni celle de la conviction ou du scepticisme de ceux qui agissent en conséquence. Si elles ne sont pas "vraies ", elles ne sont pas non plus "fausses ", car elles ne relèvent pas de l'ordre de la pensée rationnelle, mais de celui de la pensée symbolique. Il me semble à ce propos que l'on ne peut suivre Durkheim, du moins littéralement (et ma réserve porte essentiellement sur l'usage du terme vrai), lorsqu'il écrit: «Le concept qui, primitivement, est tenu pour vrai parce qu'il est collectif tend à ne devenir collectif qu'à condition d'être tenu pour vrai» $(1960,624)$.

9. D'où l'erreur de l'ethnologue qui, par souci d'empathie, prétendrait y croire lui-même et le manifester, comme le dénonce Olivier de Sardan 1988.

10. Sans nier le rôle du consensus comme fondement du partage des croyances, Crépeau (1997) souligne à juste titre qu'il est insuffisant pour rendre compte des attitudes des intéressés et attire l'attention sur la part active prise par ceux-ci dans la mise en acte de leurs croyances. 
Assurément, l'expérience cumulée des générations est déterminante pour la sauvegarde ou le rejet d'une croyance donnée et des pratiques associées. Et, par ailleurs, si des individus peuvent se plier à la norme de leur société sans éprouver pour elle une conviction enthousiaste, il est évident que la société tout entière ne saurait être cynique à son égard: il lui faut, pour «tenir ensemble ", un minimum de partage des croyances que l'on y applique du moins du système que forment ces croyances envisagées globalement, sinon de chacune prise en particulier. Il est remarquable en effet que toute société perçoit les idées qu'elle se fait du monde et de la place qu'elle y tient comme allant ensemble, comme formant un tout, même si, d'une part, elle ne les conçoit pas sous forme de système et, d'autre part, ce tout englobe des éléments empruntés ou de provenances diverses.

Mais ce n'est pas sur un quelconque jugement de "vérité ", au sens occidental du terme, que repose la validité des croyances $^{11}$ » d'une société aux yeux de ses membres, toujours conscients que d'autres sociétés ont d'autres croyances, et que chacune applique les siennes. La validité repose avant tout sur l'évidence de leur "efficacité », estimée à l'aune de la survie de la société: n'est-ce pas, d'une certaine manière, leur mise en pratique qui a permis à la société de se perpétuer jusqu'à eux dans son intégrité ? Cette notion de validité globale des croyances d'une société n'implique pas forcément, pour autant, l'efficacité effective de chacune de ses croyances prise séparément. Ainsi, tout ethnologue aura noté, sur son terrain, d'une part, le caractère machinal de certains rites et l'indifférence de certains participants à leur égard, d'autre part, la finalité éminemment pragmatique de la pratique religieuse en général. Il aura noté aussi que l'échec patent de certains rites (pour guérir un malade ou faire tomber la pluie, par exemple) est imputé au spécialiste rituel qui aura mal rempli son office, ou au commanditaire du rite qui aura commis une infraction, non à ce que l'anthropologue appelle le «système de croyances » en tant que tel. Les intéressés auront en général de nouveau recours à ce système, moyennant un appel à un autre spécialiste rituel, le rachat de l'infraction, une surenchère d'offrandes et leur présentation à un autre destinataire spirituel... Ils pourront aussi faire appel à d'autres systèmes ou d'autres types de spécialistes, sans pour autant avoir le sentiment de trahir les leurs.

11. La notion se confond ici avec celle de «traditions ", qui pourrait la remplacer dans ce paragraphe. 


\section{La distinction entre le savoir et la croyance}

Ces questions conduisent aussi tout naturellement à aborder la notion de croyance par opposition à celle de savoir. Jean Pouillon a consacré à la distinction entre ces deux notions le chapitre d'ouverture de son livre intitulé Le cru et le $s u^{12}$, dans lequel beaucoup d'anthropologues en France ont reconnu leurs préoccupations. L'auteur y dissèque la notion occidentale de croire et insiste sur la composante de doute qui s'y attache fondamentalement (si je dis « je crois ", c'est que je ne suis pas sûr) et qui le rend équivoque: «... il [le verbe "croire"] exprime tout autant l'incertitude ou l'hésitation que l'assurance» (Pouillon 1993, 21). À l'analyse, la distinction entre croyance et savoir est double. Elle porte, d'une part, sur l'attitude psychique ou mentale: la quête du savoir peut s'accompagner de certitude, la croyance ne peut ni (peut-être surtout) ne doit se débarrasser du doute ${ }^{13}$. Il est hautement significatif que l'acte de foi dans le christianisme doive s'exprimer par le verbe credo ( «je crois », et non « je sais »), compte tenu du fait qu'il implique une certaine conviction quant à l'existence de l'objet de croyance. La distinction porte, d'autre part, sur l'objet: est objet de savoir, peut-on dire en première approche, ce qui est vérifiable, passible d'un contrôle empirique indépendant du sujet; est objet de croyance ce qui est invérifiable, échappe au contrôle empirique et renvoie donc à la subjectivité, c'est-à-dire à une attitude de croyance à l'égard de l'objet en question ${ }^{14}$.

Cependant l'application de cette distinction ne va pas toujours de soi: il peut y avoir attitude de croyance sur des objets de savoir, et attitude de

12. Cet ouvrage, paru au Seuil en 1993, regroupe des versions remaniées d'articles plus anciens dont celui de 1979 cité en introduction, intitulé "Remarques sur le verbe croire ».

13. Une part de doute accompagne toute science en développement, mais ne la contredit pas dans son objectif d'établir le savoir. On doute « au nom même des exigences de la science : on ne doute que pour mieux et plus sûrement savoir, donc pour ne plus douter» (Pouillon 1993, 27).

14. On peut ici faire appel, pour illustrer cette différence, à l'exemple de l'hostie : tout le monde sait, y compris le chrétien, que c'est un disque de farine, mais le chrétien et lui seul croit que c'est le corps du Christ. C'est alors la subjectivité même qui prime, en ce qu'elle seule est capable de faire naître ce que Max Weber appelle la «motivation intérieure ", la "possession d'un état». Reprenant une phrase de saint Paul: « ... celui qui mange et boit sans discerner le corps du Seigneur mange et boit sa propre condamnation" (1Co 11,29), il mentionne la "terrible angoisse " ressentie par de nombreux chrétiens face à la communion, et la nécessité, pour la surmonter, de se mettre dans la disposition d'esprit qui rende le sacrement profitable (Weber 1996, 179-180). 
savoir sur des objets de croyance. Il peut se faire en particulier qu'un savoir soit incertain ou erroné ou encore qu'il soit déformé à des fins idéologiques, auquel cas, l'attitude à son égard devient une attitude de croyance. Ainsi, l'idée que le soleil tournait autour de la terre faisait l'objet d'une attitude de croyance de la part de l'Église chrétienne médiévale. En témoigne le fait que l'Église condamna radicalement la découverte de Copernic et sa confirmation par Galilée, fondatrices du savoir actuel sur la rotation de la terre autour du soleil.

L'histoire de la notion de vérité dans la Grèce archaïque, telle que la retrace Marcel Detienne, offrirait une illustration de la projection d'une attitude que l'on dirait aujourd'hui de savoir sur des objets de croyance. "On peut se demander, écrit l'auteur en introduction, si la vérité en tant que catégorie mentale n'est pas solidaire de tout un système de pensée [... et] aussi de la vie matérielle et de la vie sociale» (1994, 41-43). Analysant les usages archaïques du terme alètheia ( "vérité »), il remarque qu'il définit un certain type de parole et que la vérité est donc le privilège de certains types d'hommes habilités à l'énoncer (en premier lieu, les devins, les poètes et les rois de justice, puis, plus tard et différemment, les philosophes). On est bien loin des conditions de conformité à des principes logiques, d'une part, et au réel, d'autre part, qu'une culture scientifique comme la nôtre associe au sens commun de la notion de vérité (voir Detienne 1994).

Enfin, il y a des questions qui dépassent le cadre du savoir sans pour autant relever expressément de celui de la croyance. Ainsi en est-il, par exemple, remarque Denis Pelletier ${ }^{15}$, de la définition du fœtus - est-il ou non un être vivant? - , qui appelle un choix idéologique.

\section{L'attitude de «croyance»}

C'est plus tardivement dans l'histoire de l'anthropologie que l'aspect subjectif ou affectif de la croyance a retenu l'attention. Il a été abordé surtout au sein de l'anthropologie britannique. La question, lancée d'abord par Edward E. Evans-Pritchard (1956), a été reprise magistralement par Rodney Needham, qui en traite à la lumière des réflexions sur les rapports entre la langue et la pensée menées par des philosophes du langage tels que Bertrand Russell et Ludwig Wittgenstein. Son interrogation initiale portait sur la raison d'être des croyances dans la pensée de l'indigène. Que peut-on savoir

15. Séminaire du Groupe Sociétés Religions Laïcité, juin 2005. 
de son interior state ou state of mind pendant qu'il accomplit un rite? Mais tout d'abord, y croit-il vraiment ${ }^{16}$ ? Et d'ailleurs, qu'est-ce que «croire»? Dans le livre qu'il consacre à ces questions, Needham (1972) passe en revue les divers types de sentiments susceptibles de fonder un état de croyance une évidence empirique? une émotion ? un acte de volonté ? - pour aboutir au constat qu'aucun état mental ne peut être associé à la croyance ni aucun jugement de vérité ${ }^{17}$. Il reconnaît toutefois un rôle potentiel à la volonté; c'est à elle que les thérapies psychosomatiques devraient de «fonctionner » $(1972,85)$ : je crois que je vais guérir parce que je veux guérir. Conscient de ne pouvoir saisir aucun facteur décisif, Needham conclut que ce dont on dispose pour traiter du phénomène de croyance, ce sont seulement des assertions, des affirmations de croyance, qui ne disent rien sur l'attitude mentale correspondante. Au contraire, souligne-t-il, chacun sait que l'on peut faire les gestes et prononcer les paroles du croyant par pure routine ou par pur conformisme. Et il insiste: la dissimulation sociale est une nécessité (101); sans elle, la vie sociale serait impossible. En fin de compte, il n'y a pas de phénomène "croyance " à analyser, mais seulement un concept, c'est-à-dire l'usage d'un mot et des actes et postures qui l'accompagnent conventionnellement $(101,131)$. En somme, est-on tenté de répliquer, le détour par la question de l'attitude de croyance ne déboucherait-il que sur un retour à ces éléments conventionnels et accessibles à l'observation que sont le langage et les gestes rituels, si l'on veut trouver une prise pour l'analyse? Et alors, la mise en évidence des conventions qui sous-tendent l'usage des mots et la pratique des gestes n'aurait-elle pas de fortes chances de rejoindre celle des «croyances partagées» au sein de la société ?

Mais revenons à Needham. C'est un cheminement de déconstruction que poursuit cet auteur. Aussi, quand il s'avise qu'il n'y a de notion de croire

16. Question fondamentale, qui se trouve au cœur d'un livre de P. Veyne (1992) comme d'un article de R.R. Crépeau (1997). Pour cet auteur, l'usage de la notion de représentation induit indûment l'attribution d'un scepticisme généralisé aux membres des sociétés qu'étudie l'ethnologue et masque leur adhésion éventuelle aux dites représentations. En écho à cette préoccupation, j'aimerais dire ici que l'adhésion à des représentations n'empêche pas forcément la conscience que celles-ci sont des choix culturels qui ne sont pas ceux d'autres sociétés, c'est-à-dire restent de l'ordre des représentations.

17. «... as far as the supposed inner state is concerned, we have been able to discover absolutely no evidence of its existence ; and even those who are most convinced of its reality are forced in the end to conclude that they cannot give any account of it. " (Needham 1972, 123). 
comparable à la nôtre que dans les contextes sociaux imprégnés comme le nôtre par une religion universaliste, il reconnaît que sa démarche a été à son insu influencée par son éducation chrétienne et il remet en cause le postulat d'universalité de l'attitude de croyance duquel il était parti. On ne peut, ditil, faire de la croyance une catégorie naturelle de l'esprit humain. Confronté à ce qui est pour lui un constat d'échec, il renvoie la réflexion dans deux directions, d'une part, celle du langage et des actes rituels qui expriment la croyance, d'autre part, celle de l'expérience de croyance, qu'il reconnaît limitée parce qu'elle est subjective et qu'elle se heurte, comme tout ce qui confine à l'ordre de la mystique, à l'écueil de l'incommunicabilité ou de l'ineffabilité. Comparant les limites du langage avec celles du sens et de l'expérience, Needham note que «les profondeurs de l'amour, les transports de l'extase [...] sont de ceux dont nous disons qu'ils sont inexprimables, et que c'est là le signe distinctif de leur qualité ${ }^{18} »$ (Needham 1972, 229).

Il est notoire que seul le récit qui en est fait donne corps à ce qu'il est convenu d'appeler une «expérience religieuse », et que tout récit de ce genre doit se conformer au modèle implicite partagé par ceux auxquels il s'adresse pour pouvoir être accepté d'eux comme tel - ce qui amène à nouveau à s'interroger sur les mots et leur usage. Qui plus est, l'emploi de la notion d'expérientiel (c'est-à-dire ni empirique ni expérimental), en pleine expansion de nos jours dans les nouveaux mouvements religieux pour désigner l' 'expérience religieuse », vise à instaurer quelque chose comme étant de l'ordre du savoir: «je le sais parce que je l'ai vécu, je l'ai ressenti» ou «il n'y a pas à croire ou ne pas croire; cela se sent, c'est là, on le sait ». Que le sujet qui pense avoir connu une telle expérience la revendique et la formule dans les termes en cours dans le groupe suffit à la faire reconnaître comme telle par ses membres.

Par ailleurs, il est significatif que, parfois, l'on dise «croire» sur la base de l'« expérience» d'autrui, alors qu'on est conscient de n'avoir pas éprouvé soi-même cette « expérience » — autrement dit, que l'on fonde sa propre attitude de croyance sur l'énoncé de la croyance de l'autre. Voici ce qu'écrivent à ce propos les sociologues américains Bainbridge et Stark à l'issue d'une enquête menée au début des années 1960 parmi «les personnes qui prétendaient avoir eu un contact personnel direct avec des extra-terrestres» et

18. Needham ajoute en note: «It is this recognition, though, that gives plausibility to those who would impose on others with the claim that the doctrines they urge, being ultimately ineffable, are thereby of supreme value and even of unchallengeable truth. » 
autres «soucoupistes » : « ... la majorité des contactés semblaient accorder crédit aux prétentions des autres contactés et croire qu'ils étaient les seuls à simuler. [Ils...] savent parfaitement ne pas avoir de pouvoirs psychiques ou mystiques, mais continuent à penser que d'autres personnes sont d'authentiques mystiques ${ }^{19} . . . »$ De ce point de vue encore, s'imposerait un retour à l'usage des mots et à leur contexte d'emploi (sans exclure une réflexion propre sur cette sorte de croyance par procuration, qui implique que l'on attribue une valeur particulière à l'expérience subjective d'autrui et qui possède une singulière faculté de diffusion).

\section{La non-universalité de notre notion de croyance}

Il n'y a pas d'équivalent des verbes croire de l'Occident (avec leur double sens paradoxal) dans les langues des sociétés non occidentales qu'étudient les ethnologues, comme le soulignent Pouillon et Needham en divers points de leurs travaux. On ne peut, certes, inférer de l'absence d'un mot que la chose correspondante n'existe pas, et l'on pourrait admettre, à la suite de Whorf, que le concept puisse exister en tant que covert concept, remarque encore Needham (1972, 128-129). Mais c'est pour en revenir à l'idée que le seul support pour l'analyse d'un concept est l'usage du mot qui le désigne: "The phenomenon of belief consists in no more than the custom of making statements about belief» $(1972,131)$. Alors, si l'on ne peut rien dire de l'existence d'une attitude spécifique de croyance, d'une part, et si l'association linguistique entre attitude de croyance et objet de croyance n'est pas universelle, d'autre part, n'est-ce pas l'existence de cette association, ou son absence, qui devient signifiante ? Non seulement, écrit à ce sujet Pouillon, il y a du danger à appliquer à d'autres cultures une catégorie qui n'existe que dans la nôtre, mais ce danger «tient au fait que cette catégorie n'en est peutêtre pas une, même pour nous, ou du moins qu'il s'agit d'une catégorie éclatée ». C'est alors cet éclatement qui constitue, poursuit-il, "un phénomène culturel singulier » $(1993,30)$. Toutefois, cet auteur ne s'interroge pas plus avant sur ce que je préfère appeler les implications respectives de l'absence

19. Passage traduit et cité par Mayer (1985, 81, je souligne). La remarque de Bainbridge et Stark semble faire écho à celle que James G. Frazer exprime dans le tome I du Rameau d'Or: «Il résulte de là que le thaumaturge qui croit sincèrement aux pouvoirs extravagants qu'il s'attribue risque beaucoup plus d'avoir sa carrière brisée brutalement que l'imposteur délibéré [... qui a toujours] une défaite toute prête qui explique l'insuccès. " $(1988,138)$. 
ou de la présence d'une association linguistique entre objet et attitude de croyance.

\section{L'expression autonome de l'attitude de croyance}

L'absence de verbe croire ne veut évidemment pas dire l'absence de ce qu'il veut dire, ce qu'illustre du reste la facilité avec laquelle un équivalent est adopté lors de la propagation d'une religion universaliste. Ainsi, les Dangaleat, peuple tchadien qu'étudie Pouillon, utilisent des termes d'origine arabe venus avec l'islam qui veulent dire «servir ou faire confiance à ", mais qui ne sont pas réservés à l'expression du "croire » (Pouillon 1993, 30-31). Needham se demande, pour sa part, si le fait que de nombreux peuples culturellement divers emploient le sanscrit pratyaya ne prouve pas, justement, l'absence de termes analogues dans leur langue (Needham 1972, 129). Toutefois, il ne ressort pas clairement des commentaires de cet auteur si l'usage de ces termes empruntés est ou non limité à l'expression de l'attitude de croyance. Par ailleurs, un rapide survol de données comparatives donne à penser que le fait même de l'emprunt linguistique en cas d'adoption d'une religion universaliste n'est pas généralisable. La propagation peut en effet entraîner seulement l'adaptation de termes déjà existants dans la langue de réception et conservant leur sens aux côtés du sens de «croire » lié à la conversion.

\section{L'exemple mongol: la confiance ou le culte}

Il semble en particulier qu'il en soit ainsi en mongol — langue de mon propre champ de recherche - en rapport avec l'implantation du bouddhisme lamaïque, qui a débuté à la fin du XVI siècle. Plusieurs racines productives dans cette langue peuvent donner lieu à des traductions par «croire en ». La racine itgekh/itegek $\ddot{u}^{20}$ a pour sens de base: compter sur (les paroles ou la richesse de quelqu'un), avoir confiance en, se fier à, espérer en, croire en ou à (la vie future, le bouddha). La racine shütekh/sitügekü ancre la notion de

20. La première forme est translittérée de l'écriture cyrillique, la seconde de celle dite du mongol écrit. Les sens sont pris dans les dictionnaires de Lessing (1960), Cevel (1966) et Bawden (1997) pour la langue contemporaine en cyrillique, dans ceux de Kowalewski (1844-1849) et de Lessing (1960) pour la langue écrite. Ce verbe se construit avec le datif: "üge dür/burqan dur itegekü », "croire en des paroles/en le (ou les) bouddha » (Kowalewski 1844-1849, vol. 1, 291). 
croyance dans une attitude active de révérence, de vénération: soutenir, porter sur la tête, dépendre de, compter sur, se fier à, rendre un culte à, vénérer (aussi bien le bouddhisme que, aujourd'hui, la biologie). La troisième racine, bishrekh/bisirekü, assez proche de la précédente et souvent couplée à elle, a pour base l'admiration: admirer, ne jurer que par l'être ou la chose que l'on admire, croire en lui ou en elle, ou simplement respecter (par exemple les vieillards). Il convient de mentionner une autre racine encore (nominale) süseg/süzig/süsüg ( «piété, dévotion »), dont un verbe, süseglekh/ süsüglekü, signifiant "vénérer, révérer, rendre culte à » (mais non "croire en »), est dérivé, en soulignant toutefois qu'elle semble liée, sinon réservée à la foi bouddhique ${ }^{21}$.

Le substantif formé sur la première racine, itgel/itegel, veut dire "protection, patronage, fiabilité, fidélité, confiance ${ }^{22} »$; il peut s'employer aussi bien au sens de tutelle que de puissance tutélaire; il s'emploie également en couple avec d'autres noms comme espoir; il n'est pas limité au domaine religieux, mais sert à désigner la foi bouddhique dans des contextes qui rendent ce sens explicite. Un autre terme formé sur cette même racine, itgemzh/ itegemzi, s'emploie aux sens de "confiance » et de "fiabilité ». La seconde racine a deux dérivés substantifs : shüten/sitügen, qui s'applique à tout ce qui est objet de vénération (à la fois une chose sacrée, un support ou soutien sur lequel on peut compter, la représentation d'une divinité); shütleg/sitüleg, qui désigne l'acte de vénérer et s'emploie surtout en couple soit avec bishrel/ bisirel au sens de vénération, culte, soit avec shashin/shasin ${ }^{23}$ au sens de religion instituée. Quant à la racine bishrekh, elle produit, outre le substantif

21. Je n'ai, pour ma part, jamais rencontré ce terme en rapport avec le chamanisme, si ce n'est dans l'expression "mukhar süseg », littéralement " piété sans corne, piété émoussée », figée dans le sens de "superstition ».

22. Seul le dictionnaire de Kowalewski (1844-1849) mentionne expressément le sens de «protection". L'expression «itgel zhil/itegel zil», littéralement «année de protection », s'emploie pour exprimer une correspondance établie au sein du cycle calendaire des douze animaux, emprunté à l'astrologie chinoise.

23. Shashin/shasin ("précepte, doctrine "), du sanscrit çâsana, ne s'applique qu'aux religions fondées sur des écritures: "sharyn shashin» ("religion du jaune » ou bouddhisme), «lalyn shashin» ( "religion d'Allah»), «khristosyn shashin» (« religion du Christ»). L'expression «böögijn shashin» ( « religion des chamanes »), récemment apparue, est d'un usage limité à des cercles intellectuels voulant mettre le chamanisme à égalité avec ces religions. L'expression usuelle pour le désigner est «böögijn mörgöl » ( «affrontement, coup de tête de chamane»), conduite rituelle caractéristique des chamanes. 
bishrel/bisirel ( « vénération »), les adjectifs bishrem ( «admirable, digne de vénération ») et bishremtgij ( « respectueux, déférent») $)^{24}$.

Si ces trois racines - je laisse pour l'instant de côté la quatrième, süseg/ süzig, liée au bouddhisme - peuvent désigner une attitude de croyance, c'est en l'orientant dans des directions différentes. La première est la plus proche de la compréhension que nous en avons, mais lui apporte une nuance en y associant l'idée de protection, si l'on tient compte de ce sens relevé au milieu du XIX ${ }^{\mathrm{e}}$ siècle: elle exprime l'adhésion mentale à ce sur quoi l'on estime pouvoir compter pour être protégé, ou à ce qui protège et donc engendre la confiance; il s'agit donc d'une attitude de croyance fondée sur la confiance dans le pouvoir protecteur de ce en quoi l'on décide de mettre sa confiance. La seconde envisage la croyance à travers les actes concrets qui la manifestent; elle la situe sous l'angle de la pratique à laquelle elle donne lieu. Dans la perspective de la troisième racine, la croyance est une conséquence possible d'un état affectif d'admiration et de vénération ou d'un comportement plein de respect et de révérence. Ainsi, toutes ces racines sont limitées à l'expression d'attitudes mentales ou affectives de croyance, aucune ne donne un substantif correspondant à ce que nous appelons des croyances ${ }^{25}$.

S'il n'y a pas, dans les dictionnaires élaborés à partir du mongol, de termes pour dire «croyances » dérivés des verbes croire, le dictionnaire russe-mongol de Damdinsüren et Luvsandendev (1967) donne des traductions pour le terme russe qui exprime la croyance envisagée dans son contenu: verovanie ${ }^{26}$. Notons d'abord que ce terme russe est spécialisé dans ce seul sens et qu'il est dérivé d'un autre terme spécialisé dans le sens d'attitude

24. Alors que le tibétain est la langue liturgique du bouddhisme mongol, aucun de ces termes ne semble adapté de cette langue, d'après les indications fournies par ma collègue Katia Buffetrille, que je remercie. Seule la syllabe -teen de shüteen/sitügen pourrait éventuellement être rapprochée du tibétain rten/brten qui signifie «statue " en tant que support d'une divinité (mais tout rapprochement semble impossible avec brtsi 'dzin [tsidzin], brtsi sems [tsisem, « respectant, honorant »] et güs zhab [guzhab, « respect»]).

25. Ces choix sémantiques s'apparentent donc (mis à part l'absence d'emprunt dans le cas mongol) à ceux que décrit Pouillon, à propos des Dangaleat du Tchad : « ... ils ont pris [de la langue arabe] ce qui convenait à leur manière de "croire" : les termes qui désignent un comportement spécifique et une attitude mentale - rendre un culte et donner sa confiance au destinataire de ce culte - et non ceux qui renverraient à des représentations ou à des propositions définies » $(1993,31)$.

26. Le pluriel, verovanija, correspond bien à notre "croyances ". 
de «croyance» ou «foi ", vera, terme fondamental de cette racine ${ }^{27}$. Les traductions mongoles du russe verovanie, artificielles, sont révélatrices de l'embarras que cause aux auteurs l'absence de terme propre en mongol. L'une des traductions proposées est shütleg, cité plus haut, que le dictionnaire mongol de Cevel (1966) définit simplement comme acte de vénérer; le sens de "croyance " (contenu) que lui confère le dictionnaire russe-mongol constitue donc une extension qui apparaît quelque peu forcée. L'autre traduction est un néologisme formé sur la quatrième racine présentée plus haut, süseglel, qui ne figure dans aucun des dictionnaires constitués à partir du mongol. On peut penser que ce néologisme a été créé par les deux auteurs mongols du dictionnaire russe-mongol sur le modèle de la formation du terme russe verovanie, par dérivation à partir d'un terme signifiant « foi » comme le russe vera. S'il en est bien ainsi, c'est que süseg, dont on a noté plus haut le lien au bouddhisme, est, à leurs yeux, le meilleur équivalent de "foi ». Remarquons néanmoins que l'objet ou le contenu de la foi bouddhique sera désigné non par süseglel, le néologisme proposé pour «croyances » (ni par shütleg), mais par le terme nom ( «livre ») utilisé au sens de doctrine. Le bouddhisme n'est pas une "croyance».

Une remarque similaire pourrait être faite à propos des termes pour dire "croyant», "adepte» ou «dévot». Le dictionnaire russe-mongol donne, pour ces termes, des dérivés formés régulièrement sur nos quatre racines (shashind itgedeg, sütegch, bishregch, süsegten, süsegch ${ }^{28}$ ), mais absents comme tels des dictionnaires constitués à partir du mongol. Cette absence n'est pas un effet de la politique de propagande athée menée pendant des décennies par le régime communiste - leur présence dans le dictionnaire élaboré à cette époque à partir du russe suffit à le prouver. Elle est en revanche sûrement l'effet d'une habitude d'évoquer la pratique religieuse non en termes d'adhésion mentale à une religion, mais en termes de culte rendu: l'on fait référence aux rites auxquels on participe, ou au spécialiste religieux auquel on fait appel, ou encore au type de pratique rituelle que l'on accomplit.

27. Le russe ne fait donc pas peser sur la notion de croyance la même contrainte que le français ou l'anglais. Vera ne convoie pas le sens de contenu de croyance et ne s'emploie pas au pluriel.

28. Le suffixe itératif - $d e g$ signifie "qui a coutume d'accomplir l'action exprimée par le verbe ", le suffixe d'agent - $g c h$, "qui est agent de l'action exprimée par le verbe ", et le suffixe nominal -ten, "[qui est] avec ». "Shashind itgedeg ", littéralement "celui qui a l'habitude de faire confiance à la religion [bouddhique] ", serait la meilleure façon de rendre «adepte du bouddhisme». 
Autrement dit, c'est l'acte rituel qui compte: offrir une écharpe de cérémonie, sacrifier un cheval aux ancêtres, rendre un culte à une source sacrée... sans que mention soit faite de la confiance éventuelle de celui qui l'accomplit en l'instance invisible à laquelle il s'adresse ni de son état mental — conviction, indifférence, scepticisme, etc. — ni de la "vérité " éventuelle de la croyance mise en pratique. Ce qui compte aussi, c'est d'accomplir cet acte rituel de façon zöv («correcte, exacte»), et non buruu («incorrecte, contraire»). Mais on traitera familièrement de khudlaa ( «mensonges ») les «croyances » des autres ou celles que l'on ne met pas, soi, en pratique, et il faudra une périphrase explicite pour associer l'idée de vérité à une attitude de confiance : «ünen gezh itgemzhlekh ${ }^{29}$ ", «faire confiance en disant [que c'est] vrai ${ }^{30}$ ».

\section{La confiance, l'amour, la créance}

La place centrale de la notion de confiance dans les termes mongols utilisés pour dire "croire " m'incite à rappeler ici les conclusions du chapitre que Needham consacre aux étymologies des verbes croire en hébreu et dans les langues occidentales (1972, 40-50). Tous ces verbes ont leur origine dans le langage courant et leur acception religieuse est liée à l'adoption de la religion correspondante, judaïsme ou christianisme. Ainsi, les racines de «croyance» relèvent du registre de la confiance en hébreu, du registre de l'amour dans les langues anglo-saxonnes ${ }^{31}$, du registre de la créance, du fait de confier quelque chose pour en recevoir ensuite la rétribution, dans les langues romanes. Pour ce sens de credo, Needham se fonde sur l'article que Benveniste consacre à "Créance et croyance ", auquel il renvoie pour des approfondissements ${ }^{32}$.

29. Ce verbe, formé sur le nom itgemzh, appartient à la première racine, itgekh/itegekü, la plus intellectuelle.

30. La racine ünen ("vérité ») s'est considérablement développée sous le régime communiste, sous l'influence du russe pravda. Le verbe ünemshix/ünemsikü signifie «tenir pour vrai ".

31. La racine de l'anglais belief lui est commune avec l'allemand lieben.

32. Selon Benveniste, srad, l'équivalent sanscrit du credo latin, s'applique à la confiance ou plutôt à la dévotion à un dieu, sollicité d'accorder au fidèle son appui en vue qu'il obtienne la victoire; l'offrande associée au fait de mettre son srad dans un dieu comporte donc une certitude de rétribution en échange. Benveniste souligne que cette croyance n'est jamais croyance en une chose, mais une " sorte de gage ", d'enjeu matériel qui engage le sentiment personnel de confiance placé dans un être supérieur pour en recevoir un bienfait en retour, selon le principe du do ut des (Benveniste 1969, vol. 1, 171-179). 


\section{Le paradoxe de l'association linguistique entre «le croire» et ce que l'on croit}

Les registres de la confiance et de l'amour sont aussi ceux dans lesquels Max Weber avait situé l'origine de la croyance (mais par la longue voie d'autres raisonnements dont j'ai quelque scrupule à rappeler ici de façon trop schématique certains aboutissements). Cet auteur avait montré aussi que la croyance, qu'elle se présente sous la forme de la foi fondée sur la confiance ou sous celle de l'élan mystique né de l'amour, avait pour corollaire une forme de connaissance qui implique un certain «sacrifice de l'intellect»: « [Il n'existe absolument aucune religion, pour autant qu'elle reste inébranlée et agisse comme une puissance de vie, qui ne doive exiger, en un point quelconque, le "credo non quod, sed quia absurdum" : le "sacrifice de l'intellect".] (Weber 1996, 450; les crochets et italiques sont du traducteur qui, ici, résume Weber) La note du traducteur à laquelle renvoie l'appel placé après absurdum dit ceci : " "Je ne crois pas ce qui est absurde; je crois parce que c'est absurde" ; telle quelle, cette formule ne se rencontre pas chez SaintAugustin [sic], à qui Weber lui-même l'attribue [...]; on en trouverait une forme approchée chez Tertullien [...]».

Il est tentant d'examiner à la lumière de cette phrase célèbre la question du rapport entre le fait de croire et ce que l'on croit, question qui traverse cet article. En effet, cette phrase, qui se présente en quelque sorte comme une explication de l'expression "foi aveugle », implique une utilisation du même verbe croire dans ces deux sens, le second emploi étant à comprendre comme : "j'éprouve un état de croyance ", "je ressens la "motivation intérieure" ", celle dont parle Max Weber (voir la note 14 de cet article) et qui est à ses yeux la source de la «qualification religieuse». Plus précisément, cette phrase semble jouer de l'association de ces deux sens dans le même verbe, association qui, rappelons-le, caractérise l'usage de «croire» dans les langues occidentales dans lesquelles ont écrit jusqu'à nos jours la plupart des anthropologues.

J'aimerais d'abord noter, mais sans m'y attarder, que cette association linguistique donne à l'attitude de croyance la primauté logique sur l'objet de croyance, qui lui est en quelque sorte subordonné. Je voudrais plutôt avancer ici quelques propositions relatives aux implications de cette association linguistique. Elle tend à rendre l'attitude de croyance radicale, entière et systématique. Elle la rend aussi potentiellement exclusive, en fondant l'attitude de croyance sur un objet de croyance donné, ce qui peut revenir à 
exclure toute mise en cause de cet objet en lui donnant un statut de Vérité33. Ce qui peut revenir aussi à écarter a priori comme fondateur d'une attitude de croyance tout autre objet de croyance qui pourrait l'être au même titre. Cette première implication peut contribuer à expliquer que, là où existe cette association linguistique, l'attitude de croyance devienne une foi intériorisée qui se suffit à elle-même sans besoin de traduction dans des actes rituels, et dont l'affirmation exclut non le doute lui-même, mais l'expression du doute, comme pour souligner que la «motivation intérieure » en triomphe.

Une seconde implication de cette association linguistique est qu'elle tend à détacher l'attitude de croyance de toute confrontation avec la réalité. Elle peut contribuer à expliquer que l'enjeu du «croire » soit conçu en termes de «salut », renvoyé dans la «vie posthume », dans « l'au-delà ». Un tel renvoi dans une temporalité indéfinissable, située dans une sphère qui échappe au contrôle empirique, rend par avance vaine toute contestation ${ }^{34}$. Ensemble, ces deux implications peuvent contribuer à rendre compte de la force politique et identitaire d'un tel «croire» pour la société qui le partage, pour autant que le partage de croyance constitue, comme disait Pouillon, «l'armature d'une société », créant un consensus en son sein.

\section{L'absence de lien entre attitude et objet de croyance}

Il devient alors tentant d'avancer des propositions symétriques et inverses à propos des implications de l'absence d'association linguistique entre ce que l'on croit et le fait de croire, absence qui semble, quant à elle, caractériser les conceptions religieuses d'un bon nombre des sociétés qui ont fourni la matière principale des travaux d'anthropologie. Le fait que l'attitude de croyance ne soit liée par principe à aucun objet de croyance implique avant tout qu'elle n'a d'autre possibilité d'expression qu'à travers l'acte rituel auquel elle engage - et l'examen de la terminologie mongole, ci-dessus, a montré que la croyance née de la confiance n'était envisagée que sous l'angle du culte auquel elle donne lieu. Cette absence de lien rend l'attitude de croyance souple et ouverte, susceptible de se porter sur une pluralité d'objets de croyance. Elle entraîne par là même que, tout en ayant pour effet

33. Avec le corollaire que ce statut épargne à cet objet de croyance d'être soumis à démonstration.

34. J'ai eu l'occasion de développer ce report dans une temporalité inaccessible à l'expérience à propos d'un processus de construction symbolique de type messianique en Sibérie post-soviétique (voir Hamayon 2000). 
de susciter l'engagement dans l'acte rituel, l'attitude de croyance s'accommode de l'expression du doute. Elle entraîne aussi que l'acte rituel dont elle a provoqué l'exécution soit susceptible d'être soumis à l'épreuve de l'expérience - et les anthropologues savent bien à quel point les sociétés nonoccidentales qu'ils étudient attendent de leurs pratiques religieuses des "résultats » concrets. C’est pourquoi les membres de ces sociétés expriment leur posture religieuse essentiellement par référence aux rites qu'ils accomplissent et aux effets qu'ils leur attribuent.

Portée sur un objet de croyance coutumier, l'attitude de croyance - comprise, donc, au sens de ce qui motive le passage à l'acte — réactive cet objet de croyance en tant que tel et en confirme l'actualité, sans pour autant faire disparaître l'absence de certitude à son propos, comme si la question ne se posait même pas. «On fait ainsi parce qu'on l'a toujours fait en pareille occasion ", pourra-t-on dire, "cela irait mal si on ne le faisait pas ", ou « on ne sait jamais, cela peut aider; d'ailleurs cela a plutôt marché jusqu'à présent ", ou encore on recourra au fameux «je sais bien..., mais quand même» d'Octave Mannoni (1964). Aussi aimerais-je souligner que cette absence de certitude est essentielle ${ }^{35}$, ou plus précisément constitutive d'une telle attitude de croyance, en raison même de l'absence de lien avec un objet de croyance donné, mais en ce qu'elle a pour effet de pousser à l'acte, à la mise à l'épreuve de tel ou tel des objets de croyance disponibles. En ceci, l'absence de certitude se distingue radicalement du scepticisme compris comme une attitude excluant toute possibilité de certitude. Comme le dit Robert Crépeau: «en s'en tenant à cette conception [en termes de représentation], [...] seul le consensus collectif permettrait d'y adhérer. Bref, le sceptique aurait toujours raison. » $(1997,16)$

Aussi, je qualifie de dynamique le rôle que cette absence de certitude joue en tant qu'élément constitutif de l'attitude de croyance. Dynamique d'abord et surtout, mais, pour ainsi dire, en creux, parce que l'absence de certitude oblige celui qui l'éprouve à la surmonter pour s'engager dans un acte rituel ou afficher une position, à spéculer sur un objet de croyance donné. Dynamique ensuite, parce que, en cas de constat d'inefficacité de la position affichée ou du rituel engagé, elle incite à faire appel à d'autres objets de croyance, à d'autres spécialistes rituels, voire à d'autres systèmes de

35. Je rejoins ici, en partie, le propos de Grégoire Schlemmer : «le doute [...] fonde la croyance ", écrit-il dans un article (à paraître) sur l'utilisation politique de la manipulation du doute par les devins chez les Kulunge Rai du Népal. 
croyances. Dynamique enfin, parce qu'elle suscite des innovations et facilite l'adaptation au changement.

Sous ce jour, il n'est pas étonnant que, dans ces sociétés, l'absence de certitude mène à l'exécution de rituels, car ceux-ci sont des modèles de conduite considérés comme ayant fait leurs preuves. Il n'est pas étonnant non plus que la majorité de ces rituels soient non des rites liturgiques, car il n'y a pas à faire acte de dévotion, mais des rites dont l'exécution est déterminée par des opérations divinatoires et modelée par les talents personnels des spécialistes rituels, car on en espère une efficacité pratique hic et nunc. Ce sont des rituels qui n'opèrent pas par leur simple exécution, mais dont l'issue est considérée comme aléatoire et qui sont de ce fait soumis à évaluation qualitative et à sanction: la pluie est-elle tombée, l'infortune repoussée, les commanditaires satisfaits ? La contestation et l'insatisfaction entraîneront la multiplication des recours alternatifs, la transformation, l'adaptation ou l'abandon partiel du système. L'inventivité des spécialistes s'en trouvera stimulée, tout autant que le climat de rivalité qui entoure leur fonction, empêchant toute institutionnalisation de leur pouvoir et toute organisation corporatiste de leur spécialité. Il n’est pas étonnant encore que, de même que les rituels ne peuvent se prêter à la formalisation, les objets de croyance, forcément imprécis et mouvants, ne puissent donner lieu à l'établissement d'une doctrine. Il n'est pas étonnant enfin que coexistent ou s'entremêlent des éléments de systèmes religieux divers et que les individus puissent s'y livrer à plusieurs types de culte sans avoir le sentiment de trahir aucun d'entre eux ${ }^{36}$.

\section{En guise de conclusion : la mise en acte ou le pari de la croyance}

Pour mettre un terme provisoire à la réflexion proposée ici, je voudrais illustrer cette dynamique spéculative créée par l'absence de lien entre attitude et objet de croyance, par quelques exemples pris dans des cultures de Mongolie et de Sibérie qui me sont familières. Leur système religieux traditionnel, le chamanisme, accorde une place essentielle à la divination, qui illustre à deux niveaux au moins cette dynamique spéculative.

36. S'agissant des Mongols, on pourrait illustrer ce dernier propos par la célèbre ouverture d'esprit de Gengis Khan, qui a accordé sa reconnaissance à quatre religions : le bouddhisme, le christianisme, l'islam, et le taoïsme. Il est significatif qu'il ait subordonné sa reconnaissance à la condition que les intéressés prient pour sa prospérité et celle de son empire, et qu'il ait refusé toute marque d'appartenance institutionnelle comme le baptême (voir Atwood 2004). 
L'un de ces niveaux, couramment relevé, est celui où l'activité divinatoire consiste à construire un objet de croyance approprié au cas à traiter, en général un malheur à écarter. Cette construction impose d'attribuer une source à ce malheur de façon à pouvoir y porter remède en agissant sur cette source. Le principe de la réponse du chamanisme à cette question est la notion d'esprit, entité immatérielle conçue comme accessible à des relations similaires à celles des humains entre eux. Il s'agira donc, pour le chamane, de désigner un esprit responsable et de négocier avec lui l'arrêt du malheur, de proposer une hypothèse, comme dit judicieusement S.M. Shirogokoroff (1935) pour qualifier cette notion d'esprit.

C'est un autre niveau de cette dynamique spéculative qui est à mes yeux le plus fondamental. Il peut être abordé simplement à partir de la notion d' "obtenir [de] la chance» (notamment à la chasse), objet premier de la fonction chamanique. L'action rituelle consiste à « jouer»(tel est son nom ${ }^{37}$ ) en sorte de gagner une partie dont le partenaire imaginaire relève du monde des esprits. "Jouer» vise, d'une part, à donner une présence symbolique ${ }^{38}$ aux partenaires spirituels du rituel afin que la partie dont on attend la chance puisse être jouée. "Jouer» vise, d'autre part, à engager les participants ${ }^{39}$, à l'issue du rituel, à partir, par exemple à la chasse, avec l'assurance que la "chance » supposée obtenue par le chamane lors du rituel s'y concrétisera. Les participants doivent en effet donner au chamane quitus de son action, ce qu'ils ne font qu'après l'avoir obligé à recommencer plusieurs fois sa quête. Ce sont eux qui doivent formuler le résultat du lancer divinatoire que le chamane fait les yeux bandés. Une fois qu'ils ont déclaré le lancer positif, c'est à eux de prouver par une chasse ou une pêche fructueuse la validité du rituel et l'efficacité du chamane qui l'a accompli. Ainsi, le jeu rituel débouche sur la mise en acte d'une attitude de croyance qui se présente comme un pari. Il s'agit de faire comme si- pour reprendre l'expression déjà citée de Jean Pouillon, qui y ajoute d'ailleurs, de "parier sur la vérité de la croyance» (1993, 24, je souligne), je dirais plutôt de parier sur l'efficacité du rituel. Ce pari collectif a pour effet de mobiliser l'optimisme

37. L'usage de cette notion et ses implications ont été analysés dans plusieurs articles, notamment Hamayon 1998 et 1999-2000.

38. Il s'agit ici de jouer au sens théâtral, au sens de rendre présent par le mime, la mise en scène, l'évocation sonore, etc.

39. Jouer est ici envisagé dans son caractère de répétition, de simulation de l'action ou d'entraînement avant l'action. 
et la volonté des participants ${ }^{40}$. On en trouve un écho atténué dans les exclamations « jerööl boltugaj! » ( que ce vœu se réalise! ») qui ponctuent les chants de vœux ou de bénédiction clamés d'un bout à l'autre du monde mongol pour l'installation d'un nouveau couple, le premier enfilage d'un nouveau vêtement, et autres inaugurations. C'est cependant dans l'action divinatoire la plus simple et la plus courante que le volontarisme est le plus manifeste : elle consiste à lancer un objet dissymétrique, considéré comme ayant un «bon» et un «mauvais» côtés; on le lance non une fois, comme si l'on cherchait une réponse, mais jusqu'à ce qu'il tombe du «bon » côté façon de forcer le cours des choses ${ }^{41}$. Qu'il y ait une route à choisir, un mal à guérir, un danger à écarter, l'idée est latente qu'il importe de spéculer sur la réussite du lancer divinatoire pour se rendre capable de faire face à la situation.

C'est en cela que la croyance a une «utilité sociale». Raymond Aron, à qui j'emprunte cette expression, écrivait: "Il est utile que les soldats croient à la vérité des augures, que les militants croient à la victoire finale de la cause $^{42}$ » $(1967,416)$. La croyance est ce qui alimente positivement leur attitude, dans la mesure où elle est une sorte de pari sur l'avenir. Aussi le vocabulaire du jeu n'est-il, comme celui du pari, nullement incongru à son propos. Johannes Huizinga rapproche les attitudes respectives au fil de son ouvrage Homo ludens. Pour lui, le jeu et la croyance impliquent pareillement d'être «à la fois conscient et dupe » $(1951,51)$, conscients de n'être pas dans la réalité et faisant le pari de croire y être. "... la notion de jeu, écrit-il, comporte en soi la meilleure synthèse de cette unité indissoluble de croyance et de non-croyance » (52). J'aimerais proposer aussi une autre base de rapprochement, en suggérant que la place de l'absence de certitude au cœur de l'attitude de croyance peut être comparée à celle de la marge de jeu (ou latitude offerte au joueur dans l'application des règles) au cœur de l'attitude de jeu.

40. Needham ne mentionne qu'une fois la notion de pari, qu'il met en rapport avec la volonté $(1972,98)$.

41. Ce type de conduite a un aspect performatif que l'on retrouve dans les gestes de conjuration, l'ordalie, etc.

42. Il poursuit: "Sans doute est-il contraire à l'utilité sociale que les hommes avouent ne pas connaître le futur. » (ibid.) Ma formulation de cette «utilité sociale» serait un peu différente. 
Et j'aimerais enfin, sans oublier que bien d'autres aspects devraient encore être explorés ${ }^{43}$, amorcer une réponse à la question d'une participante anonyme au colloque de février 2005. Celle-ci se demandait si la part de confiance inhérente à la notion de croyance impliquait un Dieu personnel. On peut en effet penser que la pratique religieuse, dans la mesure où elle suppose l'attente d'une sorte de rétribution, s'adresse à des partenaires spirituels doués de subjectivité et d'intentionnalité, en sorte de pouvoir les impliquer dans une structure de réciprocité. Et l'on retrouverait alors l'idée, développée par Mark Anspach, qu'un pari sur l'avenir est nécessaire au fonctionnement de toute structure de réciprocité, que l'échange doit être défini «comme le fait de donner, non pas à celui qui $a$ donné, mais à celui qui va donner» $(2002,46-47)$.

\section{Références}

Anspach, M. (2002), À charge de revanche. Figures élémentaires de la réciprocité, Paris, Seuil (La couleur des idées).

Aron, R. (1967), Les étapes de la pensée sociologique, Paris, Gallimard (Tel).

ATwood, C.P. (2004), "Validation by Holiness or Sovereignty: Religious Toleration as Political Theology in the Mongol World Empire of the Thirteenth Century », International History Review, 26, p. 237-246.

Bawden, C.R. (1997), Mongolian-English Dictionary, London - New York, Kegan Paul International.

Benveniste, É. (1969), "Créance et croyance ", dans Le vocabulaire des institutions indo-européennes, Paris, Minuit, vol. 2, p. 171-179.

Certeau, M. de (1987), La faiblesse de croire, Paris, Seuil.

Cevel (Tsevel), J. (1966), Mongol khelnij tovch tajlbar' tol' [Dictionnaire raisonné de la langue mongole, en mongol], Ulaan-Baatar, Ulsyn khevlelijn khereg erkhlekh khoroo.

Coppet, D. de (1992), "De l'action rituelle à l'image, représentations comparées ", dans Philosophie et anthropologie, Paris, Centre George Pompidou (Espace International Philosophie), p. 115-130.

43. Il faudrait notamment examiner ce que cette dynamique spéculative doit au caractère métaphorique du discours religieux et aux manipulations qu'il permet sur les rapports entre ce qui métaphorise et ce qui est métaphorisé. 
CRÉPEAU, R.R. (1997), «Le chamane croit-il vraiment à ses manipulations et à leurs fondements intellectuels ? ", Recherches amérindiennes au Québec, 27/3-4, p. 7-17.

DAmdinsüren, C. et A. LuvsAndendev (1967, 1969), Oros-mongol tol'/ Russko-mongol'skij slovar', Ulaanbaatar, BNMAU-yn Shinzhlekh Ukhaany Akademi, Khel Zokhiolyn Khüreelen, 2 vol.

Detienne, M. (1994³) [1973², 1967], Les maîtres de vérité dans la Grèce archaïque, Paris, Pocket (Agora).

DuRKHEIM, É. (1960) [1912], Les formes élémentaires de la vie religieuse : le système totémique en Australie, Paris, Presses universitaires de France (Quadrige).

Evans-Pritchard, E.E. (1956), Nuer Religion, Oxford, Clarendon Press.

Frazer, J.G. (1988) [anglais 1890], Rameau d'Or, t. I: Le Roi magicien dans la société primitive / trad. par P. Sayn et H. Peyre, Paris, Robert Laffont (Bouquins).

Hamayon, R.N. (1997), "Esprit, es-tu encore là ? ", L’Homme, 37/143, p. 117-122.

— (1998), "L'épingle du jeu ", La revue du Mouvement anti-utilitariste dans les sciences sociales [MAUSS], 12: Plus réel que le réel, le symbolisme, p. 103-121.

- (1999-2000), "Des usages de "jeu" dans le vocabulaire rituel du monde altaïque ", Études mongoles et sibériennes, 30-31: Jeux rituels, p. 11-45.

- (2000), «Reconstruction identitaire autour d'une figure imaginaire chez les Bouriates post-soviétiques ", dans J.-C. AtTias, P. Gisel et L. Kaennel, dir., Messianismes. Variations sur une figure juive, Genève, Labor et Fides, p. 229-252.

HérAn, F. (1986), "Le rite et la croyance ", Revue française de sociologie, 27/2, p. 231-263.

Hervieu-Leger, D. (1997), «Croire en modernité : aspects du fait religieux contemporain en Europe ", dans F. LenOIR et Y. TARDAN-MASQuelier, dir., Encyclopédie des religions, Paris, Bayard, t. II, p. 2059-2077.

HuizingA, J. (1951) [néerlandais 1938], Homo ludens. Essai sur la fonction sociale du jeu / trad. par C. Seresia, Paris, Gallimard (Tel 130).

KoWALEWSKI, J.É. (1844-1849), Dictionnaire mongol-russe-français, Kazan, Imprimerie de l'Université, 3 vol.

Lessing, F.D. (1960) Mongolian-English Dictionary, Berkeley, University of California Press. 
LÉvi-STRAuss, C. (1957), «Le sorcier et sa magie », dans Anthropologie structurale, Paris, Plon, p. 183-203; d'abord paru dans la revue Les temps modernes, 4/41 (mars 1949) p. 385-406.

Linder, M. et J. Scheid (1993), «Quand croire, c'est faire. Le problème de la croyance dans la Rome ancienne ", Archives de sciences sociales des religions, 81, p. 47-62.

Mannoni, O. (1964), "Je sais bien... mais quand même", Les Temps modernes, 212, p. 1262-1286; aussi paru dans cet autre ouvrage du même auteur, Clefs pour l'imaginaire ou l'autre scène, Paris, Seuil (Le champ freudien), 1969, p. 9-33.

Mauss, M. (1968), Euvres, t. I: Les fonctions sociales du sacré, Paris, Minuit (Le sens commun).

Mayer, J.-F. (1985), Sectes nouvelles. Un regard neuf, Paris, Cerf.

Needham, R. (1972), Belief, Language and Experience, Oxford, Blackwell.

Olivier de Sardan, J.-P. (1988) «Jeu de la croyance et "je" ethnologique: exotisme religieux et ethno-égo-centrisme ", Cahiers d'études africaines, 111-112 (28/3-4), p. 527-540.

Pickering, W.S.F., dir. (2000), Durkheim and Representations, London I New York, Routledge.

PitT-Rivers, J. (1984-1985), "La revanche du rituel dans l'Europe contemporaine ", Annuaire de l'École Pratique des Hautes Études, section des sciences religieuses, t. XCIII, p. 41-60.

Pouillon, J. (1979), "Remarques sur le verbe “croire" ", dans M. Izard et P. SMITH, dir., La fonction symbolique. Essais d'anthropologie, Paris, Gallimard.

- (1993), Le cru et le su, Paris, Seuil.

Radcliffe-Brown, A.R. (1952), Structure and Function in Primitive Society: Essays and Addresses, London, Cohen \& West.

Schlemmer, G. (à paraître), Le scepticisme chez les Kulung (Népal), ou le doute comme opérateur de sens.

Shirokogoroff, S.M. (1935), Psychomental Complex of the Tungus, London, Kegan Paul, Trench, Trubner \& Co.

VeYne, P. (1992²) [1983], Les Grecs ont-ils cru à leurs mythes?, Paris, Seuil (Points-Essais). 
Weber, M. (1996) [1910-1920], Sociologie des religions / textes réunis, traduits et présentés par J.-P. Grossein, introduction de J.-C. Passeron, Paris, Gallimard (NRF, Bibliothèque des sciences humaines).

\section{Résumé}

Un embarras certain entoure l'usage des termes croire et croyance dans les travaux des anthropologues. L'argument développé ici porte sur l'une des causes de cet embarras: le double sens qu'ont ces termes dans les langues occidentales sous l'influence du christianisme et qui semble caractéristique des religions universalistes. Ce double sens révèle un lien implicite entre objet de croyance et attitude de croyance, la primauté logique revenant à l'attitude et l'objet lui étant subordonné. Il peut en découler que l'objet acquière un statut de Vérité et que l'attitude devienne radicale et systématique, détachée de toute confrontation avec la réalité. L'exemple de la terminologie mongole illustre, par contraste, l'absence d'un tel lien - absence qui caractérise, semble-t-il, un grand nombre de cultures nonoccidentales. En effet, les verbes mongols que l'on peut traduire par "croire» s'appliquent exclusivement à l'attitude de croyance, que les Mongols envisagent éventuellement dans son prolongement, c'est-à-dire l'action rituelle à laquelle elle donne lieu. L'absence de renvoi à des objets de croyance donnés apparaît comme une source de souplesse et d'innovation. Elle engendre une dynamique spéculative volontariste qui s'exprime surtout par les procédures divinatoires.

\section{Abstract}

The word "belief" and the verb "to believe" cause embarrassment to Western anthropologists for many reasons, particularly because they may refer either to contents (or objects) of beliefs or to the believer's state of mind (or attitude). The argument of this paper focuses on this double meaning which has been developed in Western languages under Christian influence and which seems to be specific of World religions. This double meaning reveals an implicit association between an object and the attitude towards this object-the attitude prevailing logically and the object being subordinated. This association potentially gives the object the status of absolute Truth; it also makes the attitude radical and systematic, and precludes its being confronted with realities.

By contrast, the Mongol terminology, as that of many non-Western cultures, does not imply such an association. The Mongol verbs that can be translated by "to believe" refer exclusively to the believer's attitude, along with its continuationthat is, the ritual action it leads to. The fact that it does not refer to objects of belief seems to be a source of flexibility and innovation. It generates a speculative and voluntarist dynamics that is mainly expressed in divinatory procedures. 